\title{
Le Paysage Tri-National de la Sangha
}

Maurice Tadjuidje, Barthelemy Dipapoundji et Saturnin Brice Mowawa

\section{Aperçu général sur la TNS}

Le Tri-National de la Sangha (TNS) découle d'un processus formulé lors du Sommet des Chefs d'Etats et de Gouvernements des pays de la Communauté Economique des Etats de l'Afrique Centrale (CEEAC) qui s'est tenu le 17 mars 1999 à Yaoundé (Cameroun). Ce processus a abouti à :

- la Déclaration dite de Yaoundé signée par les Etats d'Afrique Centrale pour une gestion concertée de leurs écosystèmes forestiers ;

- la naissance de la « Conférence des Ministres en charge des Forêts d'Afrique Centrale » qui plus tard deviendra la COMIFAC (Commission des Forêts d'Afrique Centrale) avec son plan de convergence adopté en 2005 ;

- la signature de l'Accord de coopération mettant en place le TNS en décembre 2000 entre les Gouvernements de la République du Cameroun, du Congo et de la République Centrafricaine ;

- I'adoption du Plan de convergence de la COMIFAC en février 2005;

- la création du Fonds Fiduciaire du Tri National de la Sangha (FTNS);

- l'engagement de la Banque Africaine de Développement (BAD) pour la COMIFAC dans la mise en œeuvre de son plan de convergence à travers létablissement du Programme de soutien à la conservation des écosystèmes du Bassin du Congo (PACEBCO);

- l'approche PACEBCo à partir de l'Unité de Gestion du Programme (UGP) basée à Yaoundé et ses antennes installées dans les six paysages écologiques du Bassin du Congo dont fait partie le TNS.

\section{Situation géographique}

Le TNS est constitué des 3 parcs nationaux contigus de Nouabalé-Ndoki (Congo), de Lobéké (Cameroun), de DzangaSangha (RCA) et de leurs zones périphériques. II sétend sur une superficie d'environ 44000 km² (Figure 1). Le climat globalement humide est marqué par des précipitations annuelles moyennes de 1500 à 2000 mm et par une courte période de sécheresse de janvier à mars.

\section{Cadre institutionnel et juridique}

Le TNS est une zone transfrontalière de conservation dans laquelle sont gérées en commun des aires protégées contigües relevant territorialement et juridiquement de chacun des trois Etats concernés, et pour laquelle les parties contractantes se sont engagées à adopter une gestion en partenariat et une réglementation commune conformément à l'Accord de coopération TNS du 7 décembre 2000 qui prévoit:

- un Comité Tri-national de Supervision et d'Arbitrage (CTSA) ;

- un Comité Scientifique Tri-national (CST) ;
- un Comité Tri-national de Suivi (CTS) ;

- un Comité Tri-national de Planification et d'Exécution (CTPE), organe à la base des activités du TNS.

\section{Population}

La population totale est de 191000 habitants. Elle est très inégalement répartie sur l'ensemble du TNS, avec un taux d'urbanisation considérablement plus élevé (dans les villes ou villages industriels) que la moyenne nationale au sein des pays du TNS. Les centres administratifs sont situés exactement sur la périphérie du TNS. L'activité économique dépend essentiellement des besoins en nourriture, viande de brousse et produits forestiers non-ligneux (PFNL). Ainsi, les revenus proviennent en grand partie de l'exploitation des ressources forestières et fauniques. Au niveau de l'aménagement du paysage, ces centres constituent une composante importante duTNS.

\section{Affectation, utilisation des terres dans le TNS}

Après les validations nationales de chaque état du TNS, le plan d'affectation des terres a fait l'objet d'une validation au niveau du TNS en novembre 2009 à Yaoundé (Cameroun). L'utilisation et l'affectation concernent:

- les parcs nationaux,

- les concessions forestières,

- les unités de gestion de la chasse (chasse sportive et à gestion communautaire),

- les zones agricoles,

- les zones de pêche

- les zones d'activités minières.

\section{Caractéristiques socio-économiques}

L'économie du TNS est entièrement fondée sur l'exploitation des ressources naturelles:

- Exploitation du bois d'œuvre,

- Développement de l'activité touristique,

- Développement de l'artisanat,

- Exploitation de la faune sauvage (viande et safari),

- Exploitation du vin de palme,

- Exploitation du raphia,

- Exploitation du poisson,

- Exploitation du diamant,

- Exploitation d'autres PFNL (gnetum, champignons, chenilles, etc.),

- Exploitation agricole.

\section{Tenure et droits des populations sur les ressources}

Le processus définissant la tenure au sein du TNS est spécifiquement régi par le droit foncier et coutumier. D'une manière générale, dans l'espace TNS, en dehors de 

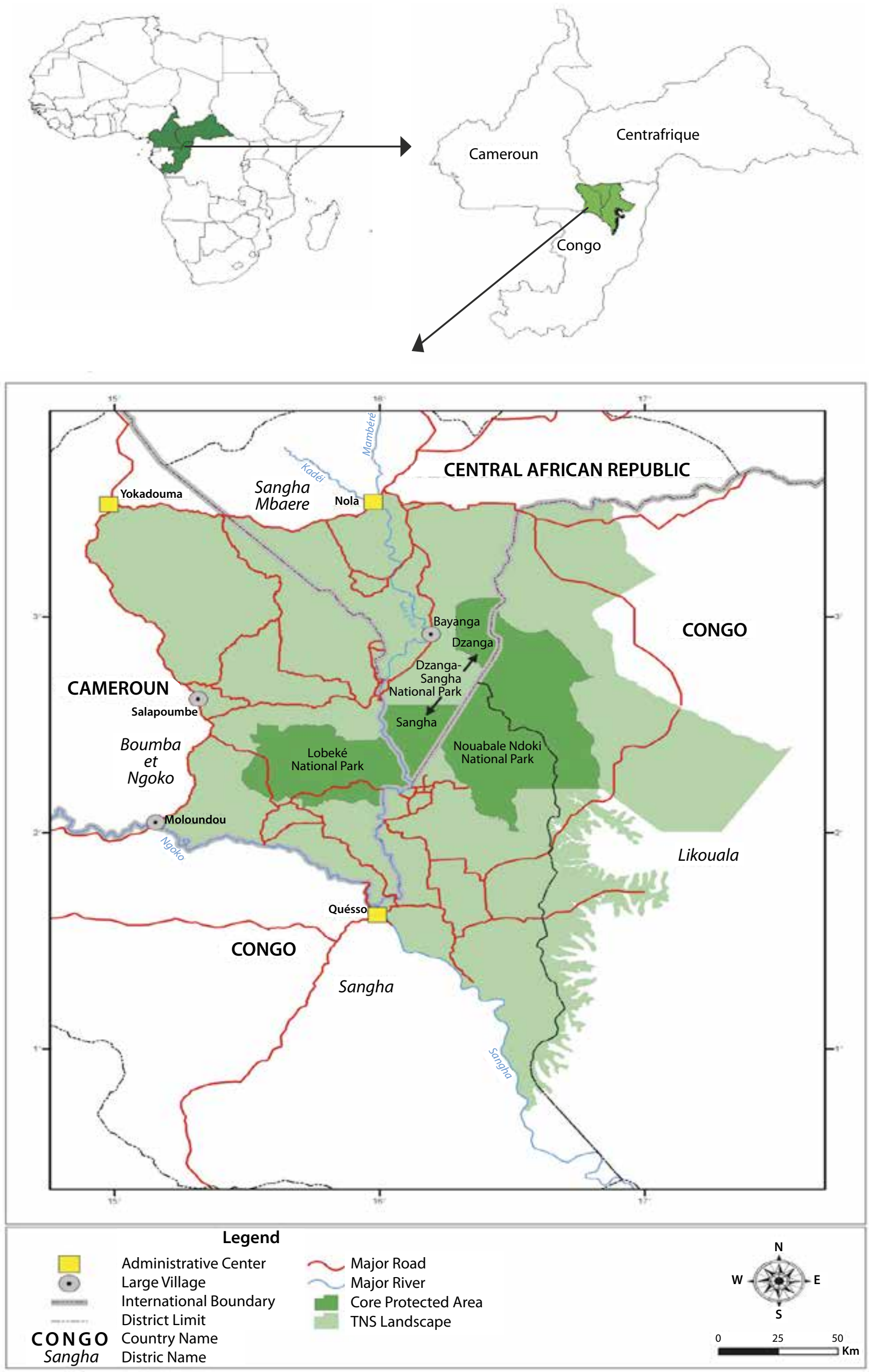

Figure 1. Le paysage TNS au sein du Bassin du Congo

Source : Zacharie NZOOH DONGMO/Project Manager Lobeke - WWF Central African Regional Programme Office (CARPO) 
la souveraineté de chaque état sur le domaine forestier permanent, il existe des domaines spécifiques réservés au droit coutumier où s'exercent les droits d'usage.

\section{Déforestation}

La déforestation dans le TNS est surtout l'œuvre des activités anthropiques liées à l'agriculture itinérante sur brûlis et à l'extraction des mines (or, diamants), ainsi qu'à l'exploitation forestière industrielle du bois. Les effets de cette dernière activité sont à ce jour contrôlés du fait du développement des outils d'aménagement et de la certification des produits forestiers. La déforestation est observée surtout autour des zones d'habitation, le long des cours d'eau (pour les extractions des mines) et des espaces cultivés longeant les routes. Selon un rapport du programme LLS de I'UICN (mars 2008), le taux de déforestation au Cameroun et en RCA est stable, variant entre 0 et $1 \%$. La FAO estime le taux de déforestation du Cameroun à 0,6\%, celui du Congo varie entre 0,1 et 0,15\%. Et en RCA, avec l'arrêt de l'exploitation forestière dans ce segment du TNS, ce taux est inférieur à $1 \%$.

\section{Biodiversité}

Le paysage forestier du TNS englobe une grande partie de la forêt dense humide guinéo-congolaise de basse altitude, riche en acajous africains et en grands mammifères. Les trois parcs constituent les principales zones de protection duTNS.

Plus de 95 \% du paysage est recouvert par la forêt, allant de la forêt semi-décidue dans le nord-ouest, à la forêt marécageuse au sud-est, avec de nombreuses clairières naturelles logées à l'intérieur et à l'extérieur des aires protégées et des Unités Forestières d'Aménagement (UFA). II héberge d'importantes populations animales classées parmi les espèces les plus menacées du continent. Les mammifères dont ceux de grande taille, tels que les éléphants de forêt, les grands primates (gorilles de plaine, chimpanzés) et les antilopes (bongo, sitatung), les petits mammifères (céphalophes, etc.) les petits primates (colobes, cercopithèques), les carnivores (léopards, chats dorés, mangoustes) et les rongeurs. L'avifaune est aussi riche de la présence de plusieurs espèces d'oiseaux résidents et migrateurs. Une des particularités du TNS est d'être arrosé du nord au sud par la Sangha, principal cours d'eau de cette région riche en icthyofaune non encore suffisamment explorée, regroupant plusieurs familles de poissons dont les Lates Niloticus couramment appelés capitaines, les Malapterudae (poissons éléctectriques), les Mormyridae (Kpété), etc.

Outre les aires protégées, le paysage est dominé par des concessions forestières qui, au cours des deux dernières décennies, ont connu une croissance exponentielle à travers l'exploitation à des fins commerciales des billes de bois dans la région.

\section{Partenaires institutionnels}

Plusieurs acteurs interviennent dans la gestion des ressources du TNS. Ce sont:
- la société civile représentée par les ONG nationales et les associations;

- les bailleurs de fonds : PACEBCo (Programme de soutien à la conservation des écosystèmes du Bassin du Congo), KfW (Banque Allemande de Développement), GIZ (Agence Internationale de Coopération Allemande), CAWHFI (Initiative pour le Patrimoine Mondial Forestier en Afrique Centrale), FTNS (Fondation pour le Tri National de la Sangha), UE (Union Européenne) ;

- $\quad$ les institutions ministérielles nationales en charge des forêts, de la faune, des aires protégées et du tourisme ;

- les ONG internationales de conservation: World Wildlife Fund for Nature (WWF), Union Internationale pour la Conservation de la Nature (UICN), Wildlife Conservation Society (WCS), etc. ;

- le secteur privé : sociétés d'exploitation forestière, minière et de safari ;

- les communautés composées des différents groupes de populations.

\section{Pressions sur les ressources}

Les diverses exploitations incontrôlées des ressources du TNS sont l'expression même de l'impact de l'homme sur la biodiversité. Le braconnage ou la pratique illégale de la chasse reste encore la menace la plus sévère, tout comme l'extraction incontrôlée du diamant, de l'or qui est une activité plus dévastatrice que l'exploitation du bois d'œuvre. Il en va de même du déboisement par les feux de forêt liés aux cultures itinérantes. La diversité biologique est sous la menace constante de l'infiltration de l'homme dans les zones protégées, cause de la réduction et/ou de la fragmentation de l'habitat et des espèces animales.

\section{Opportunités pour l'adaptation et REDD}

Les principales opportunités pour l'adaptation et la REDD dans la Tri National de la Sangha sont:

- Financements par les bailleurs de fonds des activités de développement communautaire (UE, PACEBCo, etc.) ;

- Emergence d'initiatives et d'associations liées à I'adaptation au changement climatique et à l'atténuation de ses effets ;

- Développement de mesures pour une gestion saine et durable (certification, plan d'aménagement, application des lois en matière de forêts, environnement et faune, etc.) dans les politiques et approches nationales aux différents niveaux de décision.

\section{Défis et contraintes}

Les effets du changement climatique constituent des enjeux planétaires, et le TNS ne peut s'y soustraire dès l'instant où l'amélioration des moyens utilisés affecte le bien être des populations. Inverser la tendance de la dégradation des espaces forestiers soumis à un stade très avancé de déforestation constitue un défi majeur. Dans cette optique et dans le cadre de l'adaptation au changement climatique dans certaines régions du TNS, le CIFOR a mis en place des programmes d'intervention (en organisant les communautés paysannes et en distribuant des variétés de semences améliorées de maïs à cycle court). Il est également important d'identifier et de mettre en œuvre des mesures d'atténuation du changement climatique. 


\section{Quelques images montrant l'importante biodiversité du TNS}

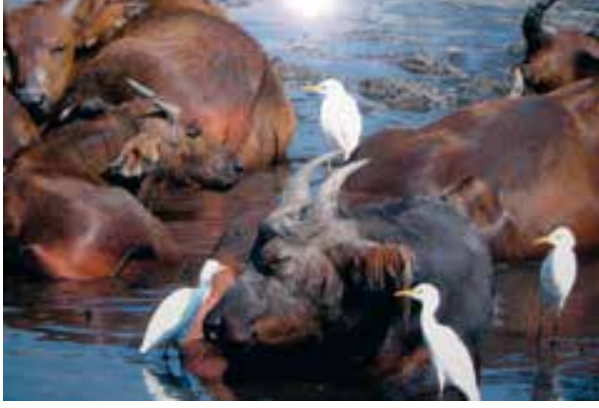

Buffles de forêts et oiseaux

(c) Thomas Breuer/Wildlife Conservation Society

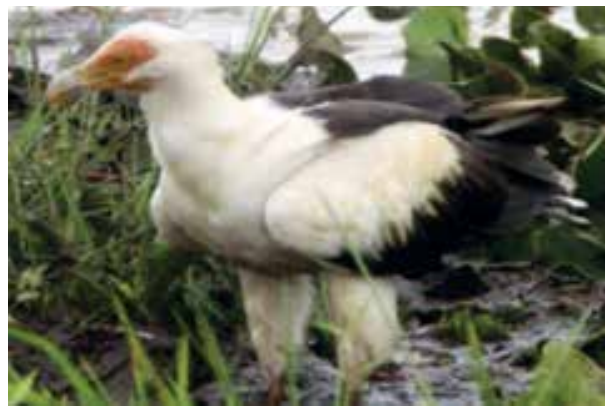

Un oiseau rapace en bordure d'une baie

(c) Thomas Breuer/Wildlife Conservation Society

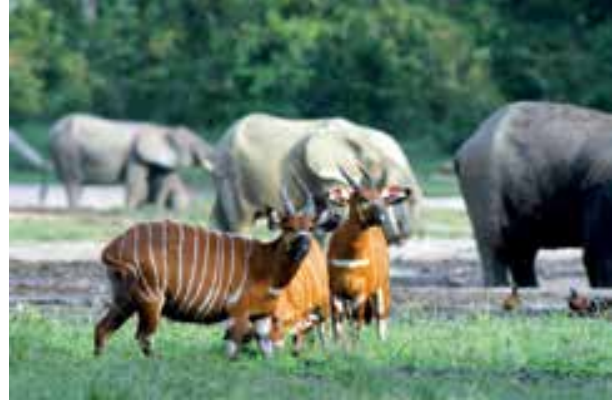

Bongo et éléphants à Dzanga (c) Andrea Turkalo

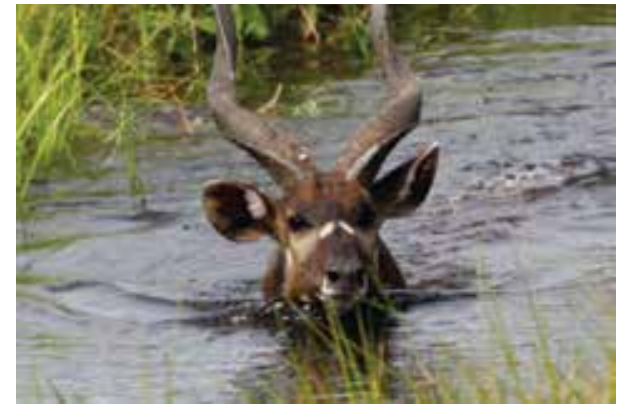

Sitatunga traversant une rivière

(c) Thomas Breuer/Wildlife Conservation Society

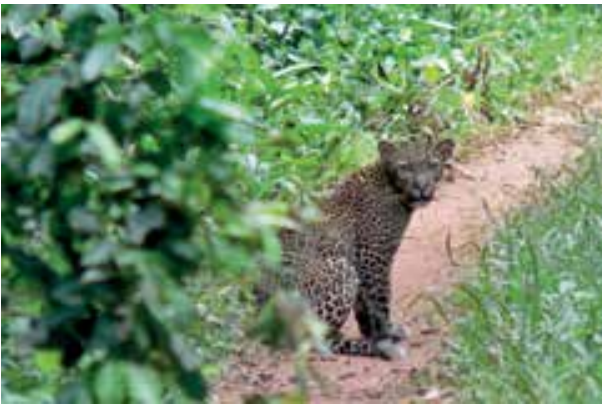

Panthère dans l'aire protégée de Dzanga Sangha (c) Philipp Roth

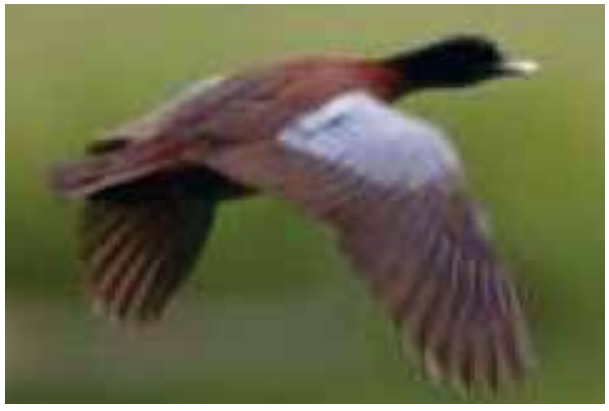

Un oiseau aquatique en vol

(c) Thomas Breuer/Wildlife Conservation Society

COBAM est mis en œuvre par le Centre pour la recherche forestière internationale (CIFOR), dans le cadre du support de la Banque africaine de développement $(B A D)$ à la Communauté économique des Etats de l'Afrique centrale (CEEAC), pour financer le Programme de soutien à la conservation des écosystèmes du Bassin du Congo (PACEBCo).
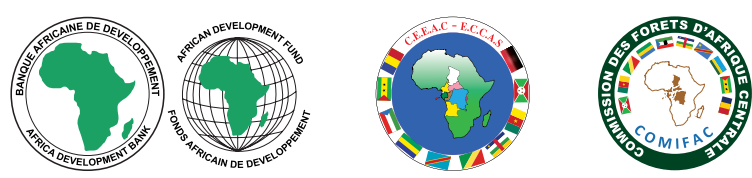

PACEBCo

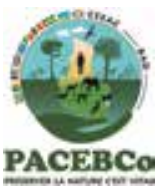

Center for International Forestry Research

CIFOR défend le bien-être humain, la conservation de l'environnement et l'équité en menant une recherche pour éclairer les politiques et les pratiques qui affectent les forêts dans les pays en développement. CIFOR est un centre de recherche du Consortium du CGIAR. Le siège du CIFOR est situé à Bogor, en Indonésie. CIFOR a également des bureaux en Asie, en Afrique et en Amérique du Sud. 